\title{
Active Fingering Instability in Tissue Spreading
}

\author{
Ricard Alert, ${ }^{1,2, *}$ Carles Blanch-Mercader, ${ }^{3,4}$ and Jaume Casademunt ${ }^{1,2}$ \\ ${ }^{1}$ Departament de Física de la Matèria Condensada, Universitat de Barcelona, Avinguda Diagonal 647, 08028 Barcelona, Spain \\ ${ }^{2}$ Universitat de Barcelona Institute of Complex Systems (UBICS), Universitat de Barcelona, 08028 Barcelona, Spain \\ ${ }^{3}$ Laboratoire Physico Chimie Curie, Institut Curie, PSL Research University, CNRS, 26 rue d'Ulm, 75005 Paris, France \\ ${ }^{4}$ Department of Biochemistry, Faculty of Sciences, University of Geneva, 30, Quai Ernest-Ansermet, 1205 Genève, Switzerland
}

(Received 28 February 2018; published 1 March 2019)

\begin{abstract}
During the spreading of epithelial tissues, the advancing tissue front often develops fingerlike protrusions. Their resemblance to traditional viscous fingering patterns in driven fluids suggests that epithelial fingers could arise from an interfacial instability. However, the existence and physical mechanism of such a putative instability remain unclear. Here, based on an active polar fluid model for epithelial spreading, we analytically predict a generic instability of the tissue front. On the one hand, active cellular traction forces impose a velocity gradient that leads to an accelerated front, which is, thus, unstable to longwavelength perturbations. On the other hand, contractile intercellular stresses typically dominate over surface tension in stabilizing short-wavelength perturbations. Finally, the finite range of hydrodynamic interactions in the tissue selects a wavelength for the fingering pattern, which is, thus, given by the smallest between the tissue size and the hydrodynamic screening length. Overall, we show that spreading epithelia experience an active fingering instability based on a simple kinematic mechanism. Moreover, our results underscore the crucial role of long-range hydrodynamic interactions in the dynamics of tissue morphology.
\end{abstract}

DOI: 10.1103/PhysRevLett.122.088104

The spreading of epithelial monolayers by collective cell migration is crucial for tissue morphogenesis, wound healing, and tumor progression. Both in vivo and in vitro, multicellular protrusions called epithelial fingers often appear at the front of spreading tissues (Fig. 1) [1-11]. This epithelial fingering resembles the viscous fingering that occurs via the Saffman-Taylor instability when a viscous fluid displaces a more viscous one $[12,13]$. However, the mechanisms of these two phenomena must be different because epithelial monolayers are more viscous than the fluid that they displace. Hence, several models of epithelial fingering have been proposed [3].

To induce finger formation, some models directly implement leader cells with a distinct behavior, either via special particles [14] or via a dependence of the magnitude of cell motility forces on the curvature of the tissue front $[15,16]$. Other models recapitulate epithelial fingering by introducing alignment between cell motility forces and the tissue velocity field [17]. These models predict a moving front to be stable and a nonmoving front to exhibit an instability with an unbounded growth rate for a number of finite wavelengths $[18,19]$. Fingers were also observed in the numerical solution of other continuum models of spreading epithelia, either treated as active polar fluids [20] or as active nematics with cell proliferation [21]. Recently, fingering was also found in a parameter range of an active vertex model [22]. Finally, interface undulations can emerge from the coupling of chemotactic fields to the mechanics of epithelial spreading [23-26].
Despite the many efforts, the physical mechanism of the fingering instability in epithelia remains a matter of debate. Here, we address this problem by means of a continuum active polar fluid model for epithelial spreading. The model includes hydrodynamic interactions through the tissue, and it implements neither leader-cell behavior nor alignment between cellular traction forces and the flow field. Yet, we analytically predict a long-wavelength instability of the moving front that explains epithelial fingering. The instability is based on a generic kinematic mechanism, namely, the front acceleration associated to a fixed velocity gradient. In spreading epithelia, the velocity gradient is imposed by active traction forces at the edge of the viscous cell monolayer. The fastest-growing mode has a finite wavelength, typically a few hundred micrometers, consistent with the measured finger spacing [11]. This characteristic wavelength is selected by the long-range hydrodynamic interactions in the tissue, which are either

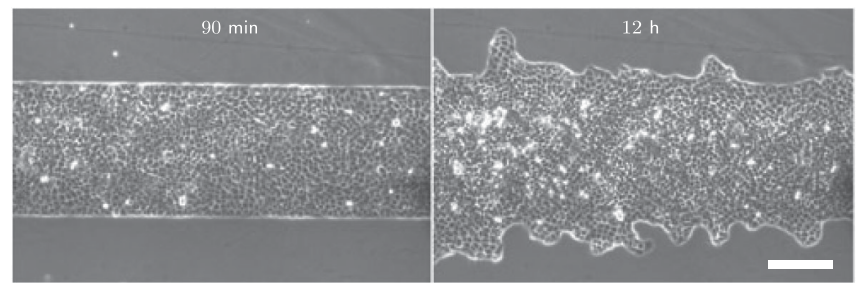

FIG. 1. Fingering in epithelial spreading. Scale bar, $200 \mu \mathrm{m}$. Adapted from [6] with permission from Pascal Silberzan. 
limited by the tissue size or screened by cell-substrate friction forces. The model also shows that intercellular contractility stabilizes short-wavelength perturbations of the tissue boundary. The stabilizing effect of contractility is typically stronger than that of tissue surface tension. Globally, our analysis shows how, as a result of the flows induced by the traction force field, a morphological instability may naturally take place in a spreading cell monolayer. Leader cells could then appear upon the onset of the instability, influencing finger development.

Model.-We base our analysis on a continuum active polar fluid model of epithelial spreading, which is, thus, described in terms of a polarity field $\vec{p}(\vec{r}, t)$ and a velocity field $\vec{v}(\vec{r}, t)$ [27-29]. We neglect cell proliferation and the bulk elasticity of the monolayer, which eventually limit the spreading process $[6,17,30-32]$. Tissue spreading is primarily driven by the traction forces exerted by cells close to the monolayer edge, which polarize perpendicularly to the edge by extending lamellipodia towards free space. In contrast, the inner region of the monolayer remains essentially unpolarized, featuring much weaker and transient traction forces $[27,28]$. Hence, we take a free energy for the polarity field that favors the unpolarized state $p=0$ in the bulk, with a restoring coefficient $a>0$, and we impose a normal and maximal polarity as a boundary condition at the tissue edge. In addition, the polar free energy includes a cost for polarity gradients, with $K$ the Frank constant of nematic elasticity in the one-constant approximation [33]. Altogether,

$$
F=\int\left(\frac{a}{2} p_{\alpha} p_{\alpha}+\frac{K}{2}\left(\partial_{\alpha} p_{\beta}\right)\left(\partial_{\alpha} p_{\beta}\right)\right) d^{3} \vec{r} .
$$

We assume that the polarity field is set by flow-independent mechanisms, so that it follows a purely relaxational dynamics, and that it equilibrates fast compared to the spreading dynamics [34]. Hence, $\delta F / \delta p_{\alpha}=0$, which yields

$$
L_{c}^{2} \nabla^{2} p_{\alpha}=p_{\alpha}
$$

where $L_{c}=\sqrt{K / a}$ is the characteristic length with which the polarity modulus decays from $p=1$ at the monolayer edge to $p=0$ at the center.

Then, force balance imposes

$$
\partial_{\beta} \sigma_{\alpha \beta}+f_{\alpha}=0
$$

where $\sigma_{\alpha \beta}$ is the stress tensor of the monolayer, and $f_{\alpha}$ is the external force density acting on it. Since tissue spreading occurs over time scales of several hours (Fig. 1), we neglect the elastic response of the tissue [34]. Thus, we relate tissue forces to the polarity and velocity fields via the following constitutive equations for an active polar fluid [61] (see discussion and justification in Ref. [34]):

$$
\begin{aligned}
\sigma_{\alpha \beta} & =\eta\left(\partial_{\alpha} v_{\beta}+\partial_{\beta} v_{\alpha}\right)-\zeta p_{\alpha} p_{\beta}, \\
f_{\alpha} & =-\xi v_{\alpha}+\zeta_{i} p_{\alpha} .
\end{aligned}
$$

Here, $\eta$ is the effective monolayer viscosity, and $\xi$ is the cell-substrate friction coefficient. Respectively, $\zeta<0$ is the active stress coefficient accounting for the contractility of polarized cells, and $\zeta_{i}>0$ is the contact active force coefficient accounting for the maximal traction stress exerted by polarized cells on the substrate, $T_{0}=\zeta_{i} h$, with $h$ the monolayer height.

Stability of the tissue front.-To study the stability of the advancing front, we consider a rectangular monolayer typical of in vitro experiments (Fig. 1). Thus, the reference state is the flat front solution with $\vec{p}=p_{x}^{0}(x) \hat{x}$ and $\vec{v}=$ $v_{x}^{0}(x) \hat{x}$ [34] [dashed lines in Fig. 2(a)]. In addition to a maximal normal polarity at the edges, we impose stressfree boundary conditions. For an interface of arbitrary shape, $\vec{p}(x= \pm L)=\hat{n}_{ \pm}$, and $\left.\sigma \cdot \hat{n}_{ \pm}\right|_{x= \pm L}=\overrightarrow{0}$, respectively, where $\hat{n}_{ \pm}$is the normal unit vector of the top and bottom interfaces. The tissue width $L$ changes according to $d L / d t=\left.\vec{v} \cdot \hat{n}\right|_{x=L}$. Then, motivated by experimental observations (Fig. 1), we introduce peristaltic smallamplitude perturbations of the flat interface, namely, those that modify the monolayer width [Fig. 2(a)]: $L(y)=$ $L_{0}+\delta L(y)$. From a linear stability analysis [34], we obtain that the growth rate $\omega(q)$ of such perturbations is always real, so that no oscillatory behavior is expected. However, the growth rate evidences a long-wavelength instability of the monolayer front. Moreover, the fastest-growing perturbation has a finite wavelength [Fig. 2(b)]. In the following, we analyze the contribution of the different forces to the instability, which allows us to single out its physical mechanism.

Traction forces.-First, we consider a limit case with neither intercellular contractility nor cell-substrate friction, $\zeta, \xi \rightarrow 0$ [27]. In addition, we also consider that the width of the polarized boundary layer of cells is much smaller than the total tissue width, $L_{c} \ll L_{0}$, which is generally the case in experiments $[27,28]$. In this limit, since active forces are concentrated at the narrow boundary layer, most
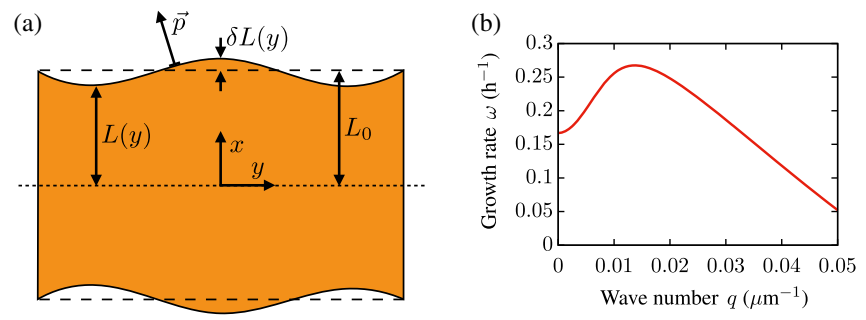

FIG. 2. Instability of the monolayer front. (a) Sketch of the peristaltic perturbations. Dashed lines indicate the flat, unperturbed interface. The dotted line indicates the symmetry axis of the monolayer. (b) Growth rate of the perturbations. Parameter values are in Table I. 
of the tissue behaves as a passive viscous fluid, for which $\partial_{x} \sigma_{x x} \approx 0$ and $\sigma_{x x} \approx 2 \eta d v_{x} / d x$. Therefore, the stress is uniform throughout most of the tissue, with a value given by the stress accumulated across the boundary layer, namely, $\sigma_{x x} \approx T_{0} L_{c} / h$. Consequently, the velocity gradient is also fixed and uniform, and hence, the spreading velocity $v_{x}\left(L_{0}\right)=V_{0}=d L_{0} / d t$ reads

$$
V_{0} \approx \frac{T_{0} L_{c}}{2 \eta h} L_{0} \equiv \frac{L_{0}}{\tau},
$$

where we have used that $v_{x}(0)=0$. This result means that, due to the sole action of a constant traction force, the flat front accelerates, consistent with measurements $[6,62]$ and with the size dependence of tissue wetting [28]. Consequently, the $q=0$ perturbation mode is unstable, $\omega(q=0)=\tau^{-1}>0$, since any uniform displacement of the advancing front makes it depart from its original velocity. Thus, the instability mechanism is kinematic in nature: The advanced regions of the front move faster than the trailing regions [Fig. 2(a)]. Thereby, traction forces contribute to destabilize perturbations of all wavelengths [Fig. 3(a)]. Therefore, since other forces such as surface tension stabilize short-wavelength perturbations, the tissue front experiences a long-wavelength instability.

Viscous stresses.-The kinematic mechanism explains why long-wavelength modes are unstable. However, it does not explain why the most unstable mode occurs at a finite
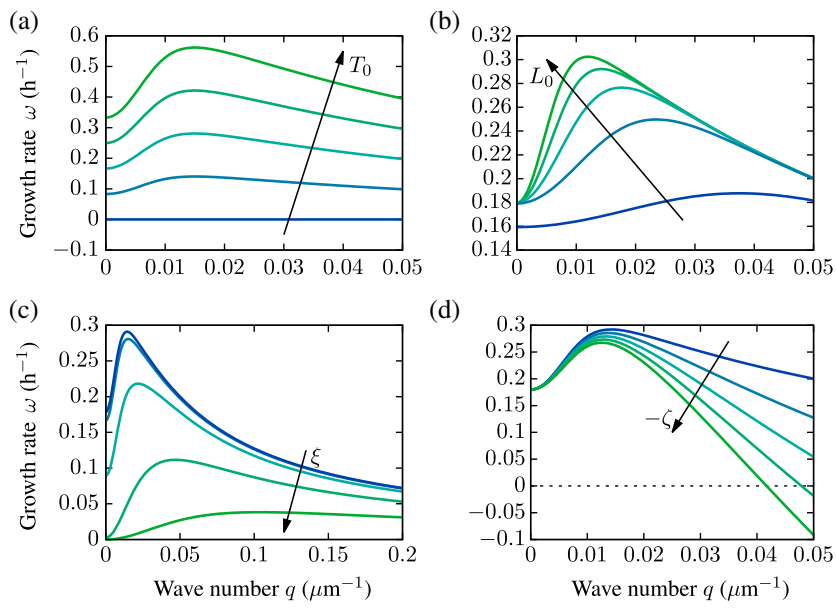

FIG. 3. Contributions to the instability. Growth rates of shape perturbations varying the values of different model parameters. Excluding the varied parameter, other parameter values are in Table I except for $\xi, \zeta \rightarrow 0$. (a) Traction forces completely destabilize the monolayer front. For this plot, $T_{0}=0,0.25$, $0.5,0.75,1 \mathrm{kPa}$. (b) Long-range transmission of viscous stresses selects the fastest-growing mode. For this plot $L_{0}=50,100,150$, 200, $250 \mu \mathrm{m}$. (c) Cell-substrate friction screens hydrodynamic interactions to limit the wavelength of the fingering pattern. For this plot, $\xi=10,10^{2}, 10^{3}, 10^{4}, 10^{5} \mathrm{~Pa} \mathrm{~s} / \mu \mathrm{m}^{2}$. (d) Contractility stabilizes short-wavelength perturbations of the monolayer front. For this plot, $-\zeta=0,10,20,30,40 \mathrm{kPa}$. wavelength [Fig. 3(a)]. In fact, the existence of a peak in the growth rate is due to the transmission of viscous stress across the monolayer. In the so-called wet limit $\lambda=$ $\sqrt{\eta / \xi} \gg L_{0}$, corresponding to $\xi \rightarrow 0$, viscous stresses transmit through the entire monolayer. Thus, a given perturbation of the front generates a flow perturbation that penetrates a distance of the order of its wavelength, $\pi / q$, into the monolayer. At the monolayer edge, the stress-free boundary condition imposes $\delta \sigma_{x x}( \pm L)=\mp \partial_{x} \sigma_{x x}^{0}\left( \pm L_{0}\right) \delta L$. Hence, since $\delta \sigma_{x x}=2 \eta \partial_{x} \delta v_{x}$ in the absence of contractility $(\zeta \rightarrow 0)$, the gradient of the velocity perturbation profile is fixed at the boundary, being positive (negative) for advanced (trailing) regions of the tissue front. Therefore, flow perturbations further destabilize the flat front in a wavelength-dependent manner.

For short wavelengths, $\pi / q<L_{0}$, the penetration distance of flow perturbations is shorter than the tissue width. Thus, since the slope of velocity perturbations at the interface is fixed, the longer the wavelength, the larger the interfacial velocity perturbation [Fig. 4(a)]. Hence, the growth rate increases with the wavelength [Fig. 3(a)]. In contrast, for long wavelengths, $\pi / q>L_{0}$, the penetration distance of flow perturbations is longer than the tissue width. Thus, in this case, the decay of flow perturbations becomes nearly linear, with a slope that decreases with increasing wavelength. Consequently, perturbations of longer wavelength feature a smaller interfacial velocity perturbation [Fig. 4(a)], and hence, they are less unstable [Fig. 3(a)]. In conclusion, in the absence of cell-substrate friction forces, the finite width of the monolayer limits the
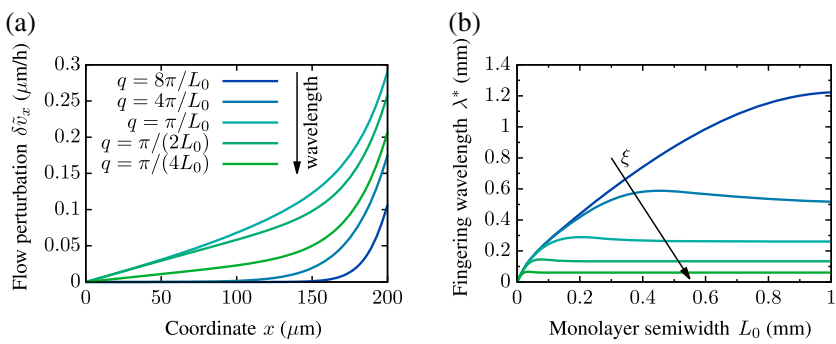

FIG. 4. Screening of tissue flows. (a) Flow perturbations induced by short-wavelength shape perturbations $\left(q>\pi / L_{0}\right)$ penetrate a distance given by their wavelength. Thus, the interfacial velocity perturbation increases with wavelength. In contrast, the penetration of flow perturbations induced by longwavelength shape perturbations $\left(q>\pi / L_{0}\right)$ is limited by the tissue width $2 L_{0}$, which entails a decrease of the interfacial velocity perturbation. Consequently, shape perturbations with a wavelength that matches the monolayer width $\left(q \sim \pi / L_{0}\right)$ feature the fastest growth [Fig. 3(b)]. Parameter values are in Table I except for $\xi, \zeta \rightarrow 0$. (b) The wavelength of the fastest-growing mode, $\lambda^{*}$, is proportional to the monolayer semiwidth $L_{0}$ if $L_{0} \lesssim \lambda$, with $\lambda=\sqrt{\eta / \xi}$ the hydrodynamic screening length. For wider monolayers, the selected wavelength is size independent, becoming proportional to $\lambda$. Parameter values are in Table I except for $\zeta \rightarrow 0$, and $\xi=10,10^{2}, 10^{3}, 10^{4}, 10^{5} \mathrm{~Pa} \mathrm{~s} / \mu \mathrm{m}^{2}$. 
range of hydrodynamic interactions in the tissue, thus giving rise to the peak in the growth rate at $q^{*} \sim \pi / L_{0}$ [Fig. 3(b)].

Cell-substrate friction forces.-Cell-substrate friction screens the transmission of viscous stresses over distances larger than $\lambda=\sqrt{\eta / \xi}$. Consequently, the peak of the growth rate occurs at $q^{*} \sim \pi / \lambda$ if $\lambda \lesssim L_{0}$ [Fig. 3(c)]. Thus, for sufficiently strong friction, the fingering wavelength is given by the hydrodynamic screening length $\lambda$, instead of the monolayer width $L_{0}$ [Fig. 4(b)]. We estimate $\lambda \sim 0.5 \mathrm{~mm}$ (Table I). Therefore, the crossover from a viscosity-dominated to a friction-dominated regime of monolayer spreading and fingering should be observable in usual in vitro experiments.

Surface tension.-The monolayer edge presents a surface tension $\gamma$, namely, the work per unit area required to expand it. For a curved interface, surface tension gives rise to a normal force: $\left.\hat{n}_{ \pm} \cdot \boldsymbol{\sigma} \cdot \hat{n}_{ \pm}\right|_{x= \pm L}=-\left.\gamma \vec{\nabla} \cdot \hat{n}_{ \pm}\right|_{x= \pm L} \approx$ $\pm \gamma d^{2} \delta L / d y^{2}$. Then, the expansion of the growth rate at long wavelengths reads

$$
\omega(q) \approx \frac{1}{\tau}+\left[\frac{1}{3 \tau}-\frac{\gamma}{2 \eta L_{0}}\right]\left(q L_{0}\right)^{2}+\mathcal{O}\left(\left(q L_{0}\right)^{4}\right),
$$

with $\tau=2 \eta h /\left(T_{0} L_{c}\right)$. This expression reveals the existence of a critical size $L_{\gamma} \approx 3 \gamma h /\left(T_{0} L_{c}\right)$ above which the growth rate curves upwards at $q \rightarrow 0\left[\omega^{\prime \prime}(0)>0\right]$, hence, exhibiting the aforementioned peak at a finite wavelength. Alternatively, if $\gamma>\gamma^{*} \approx T_{0} L_{c} L_{0} /(3 h)$, surface tension prevents the hydrodynamic selection of a finite fingering wavelength, which is then only limited by the length of the tissue front.

The surface tension of the monolayer could be due to actin cables found along its edge, particularly along the sides of epithelial fingers $[6,9,10]$. Traction force measurements suggest that the tension of such cables is $\gamma \sim$ $0.2 \mathrm{mN} / \mathrm{m}$ [10], lower than typical surface tensions of cell aggregates, $\gamma \sim 1-10 \mathrm{mN} / \mathrm{m}$ [65-68]. Combining these value ranges with $T_{0}=0.2-0.8 \mathrm{kPa}$ and typical values of $h$ and $L_{c}$ (Table I), the critical monolayer width for fingering is $L_{\gamma} \sim 0.3-10 \mu \mathrm{m}$. Therefore, we expect

TABLE I. Estimates of model parameters.

\begin{tabular}{lll}
\hline \hline Symbol & \multicolumn{1}{c}{ Description } & \multicolumn{1}{c}{ Estimate } \\
\hline$L_{0}$ & Monolayer half-width & $200 \mu \mathrm{m}$ \\
$h$ & Monolayer height & $5 \mu \mathrm{m}[28,63]$ \\
$L_{c}$ & Nematic length & $25 \mu \mathrm{m}[27,28]$ \\
$T_{0}$ & Maximal traction & $0.5 \mathrm{kPa}[27,28]$ \\
$-\zeta$ & Intercellular contractility & $20 \mathrm{kPa}[28]$ \\
$\xi$ & Friction coefficient & $100 \mathrm{~Pa} \mathrm{~s} / \mu \mathrm{m}^{2}[64]$ \\
$\eta$ & Monolayer viscosity & $25 \mathrm{MPa} \mathrm{s}[27,28]$ \\
$\lambda$ & Hydrodynamic screening length & $0.5 \mathrm{~mm}(\sqrt{\eta / \xi})$ \\
\hline \hline
\end{tabular}

surface tension not to play a major role in the fingering instability in monolayers of typical widths $L_{0} \sim 0.1-1 \mathrm{~mm}$.

Intercellular contractility.-Because it decreases the spreading velocity, intercellular contractility has an additional stabilizing effect on the monolayer front [Fig. 3(d)]. We discuss the effects of a uniform contractility in [34]. Here, we consider an intercellular contractility $-\zeta$ concentrated at the polarized boundary layer, which has a sizeindependent contribution to the spreading velocity,

$$
V_{0} \approx \frac{T_{0} L_{c}}{2 \eta h} L_{0}+\frac{L_{c}}{2 \eta}\left[\frac{\zeta}{2}-\frac{T_{0} L_{c}}{h}\right] .
$$

Consequently, in the limit $L_{c} \ll L_{0} \ll \lambda$, this contractility has no impact on the growth rate of the uniform mode, $\omega(0)=\tau^{-1}$, but it contributes a stabilizing quadratic term to the long-wavelength expansion of the growth rate,

$$
\omega(q) \approx \frac{1}{\tau}+\left[\frac{1}{3 \tau}+\frac{\zeta L_{c}^{2}}{8 \eta L_{0}^{2}}\right]\left(q L_{0}\right)^{2}+\mathcal{O}\left(\left(q L_{0}\right)^{4}\right) .
$$

Thus, as surface tension, contractility defines a critical size $L_{\zeta} \approx \sqrt{-3 \zeta L_{c} h /\left(4 T_{0}\right)}$ above which the growth rate features a finite-wavelength peak. Alternatively, if $-\zeta>-\zeta^{*} \approx$ $4 T_{0} L_{0}^{2} /\left(3 h L_{c}\right)$, contractility suppresses the hydrodynamic selection of a finite fingering wavelength.

For typical contractilities, $-\zeta \sim 1-50 \mathrm{kPa}$ [28], the critical monolayer width for fingering is $L_{\zeta} \sim 10-100 \mu \mathrm{m}$, where we have used $T_{0}=0.2-0.8 \mathrm{kPa}$ and estimates for $h$ and $L_{c}$ (Table I). Therefore, we do not expect contractility to prevent fingering wavelength selection. However, our estimates give $L_{\gamma}<L_{\zeta}$, indicating that contractility typically dominates over surface tension in stabilizing shortwavelength shape perturbations. Thus, the competition between the destabilizing effect of traction forces and the stabilizing effect of intercellular contractility defines the band of unstable modes.

Uniformly polarized tissues.-To consider monolayers with bulk polarity [63], we analyze the morphological stability of a uniformly polarized monolayer [34]. In this case, in the absence of contractility, the spreading velocity is constant: $V_{0}=T_{0} /(\xi h)$. Hence, the $q=0$ mode is marginally stable, $\omega(q=0)=0$. Moreover, contractility stabilizes it, $\omega(0)<0$. Nevertheless, the viscous effects discussed above still give rise to a peak of the growth rate at a finite wavelength. Therefore, even though uniformly polarized tissues do not feature an accelerating front, they still exhibit a fingering instability for sufficiently small contractility [34].

Conclusions.-Motivated by the observation of fingerlike protrusions during the spreading of epithelial monolayers, we studied the stability of the advancing front. Modeling the cell monolayer as an active polar fluid, we showed that active traction forces are responsible for a long-wavelength instability of the monolayer front. Several 
features distinguish this instability from previous proposals. First, it is generic; it takes place for any value of the active traction force. Second, the wavelength of the fingering pattern is selected by the range of hydrodynamic interactions in the tissue. Third, active intercellular forces stabilize short-wavelength perturbations, typically dominating over surface tension effects.

Our analysis identifies the physical mechanism of the instability. Cellular traction forces at the monolayer edge set the velocity gradient in the spreading monolayer. Hence, under the same traction force, a larger monolayer spreads faster [28]. Consequently, when the monolayer front is perturbed, the protruding regions of the interface advance faster than the trailing regions, thus, making the perturbation grow. Therefore, the instability is based on a simple kinematic mechanism, which takes place generically in viscous fluids that sustain a fixed velocity gradient in the direction of spreading. In particular, the same morphological instability should occur in the socalled squeeze flow [69], in which an incompressible fluid is forced to spread by decreasing the gap between two plates. In this case, under perfect slip conditions at the plates, the rate of gap reduction sets the fixed velocity gradient.

Regarding spreading epithelia, we conclude that neither leader-cell behavior nor regulation of cell motility by curvature or by chemotactic fields are necessary for the fingering instability. Therefore, our results are consistent with the emergence of leader cells concomitantly with finger growth $[6-8,10,11]$. However, the viscous rheology of the monolayer is essential for the instability. On the one hand, it underpins the velocity gradient that renders the interface unstable, and on the other hand, it enables wavelength selection for the fingering pattern. Concomitant with the fingering instability, shear stresses give rise to flows transversal to the spreading direction, which might lead to the swirls observed in experiments [6,7]. Finally, in addition to explaining fingering in tissue spreading, our results also account for the morphological instability recently observed during tissue dewetting [28].

Our predictions, such as the absence of a traction force threshold for the instability and whether the fingering wavelength is given by either the monolayer width or the screening length $\lambda=\sqrt{\eta / \xi}$, are experimentally testable. Indeed, consistent with our result, recent work has shown that the finger spacing is an intrinsic quantity that coincides with the stress correlation length [11]. To further test our predictions, future experiments could perturb active cellular forces, cell-cell and cell-substrate adhesion, and vary monolayer width.

Our findings illustrate how hydrodynamic interactions impact tissue morphodynamics. In particular, we propose that epithelial fingering can naturally arise from a generic morphological instability in a fluid film driven by interfacial active forces. Thus, our results showcase the relevance of interfacial instabilities in driven [70-72] and active [73-79] fluids for tissue spreading.

We thank Xavier Trepat for a critical reading of the manuscript, and the members of his lab for discussions. We thank Jordi Ortín for discussions. R. A. acknowledges support from Fundació "La Caixa". R. A. and J. C. acknowledge the MINECO under Project No. FIS201678507-C2-2-P and Generalitat de Catalunya under Project No. 2017-SGR-1061.

*Present address: Princeton Center for Theoretical Science and Lewis-Sigler Institute for Integrative Genomics, Princeton University, Princeton NJ 08544, USA. ricard.alert@princeton.edu

[1] S. R. K. Vedula, A. Ravasio, C. T. Lim, and B. Ladoux, Collective cell migration: a mechanistic perspective, Physiology 28, 370 (2013).

[2] T. B. Saw, S. Jain, B. Ladoux, and C. T. Lim, Mechanobiology of collective cell migration, Cell. Mol. Bioeng. 8, 3 (2015).

[3] V. Hakim and P. Silberzan, Collective cell migration: a physics perspective, Rep. Prog. Phys. 80, 076601 (2017).

[4] B. Ladoux and R.-M. Mège, Mechanobiology of collective cell behaviours, Nat. Rev. Mol. Cell Biol. 18, 743 (2017).

[5] T. Omelchenko, J. M. Vasiliev, I. M. Gelfand, H. H. Feder, and E. M. Bonder, Rho-dependent formation of epithelial "leader" cells during wound healing, Proc. Natl. Acad. Sci. U.S.A. 100, 10788 (2003).

[6] M. Poujade, E. Grasland-Mongrain, A. Hertzog, J. Jouanneau, P. Chavrier, B. Ladoux, A. Buguin, and P. Silberzan, Collective migration of an epithelial monolayer in response to a model wound, Proc. Natl. Acad. Sci. U.S.A. 104, 15988 (2007).

[7] L. Petitjean, M. Reffay, E. Grasland-Mongrain, M. Poujade, B. Ladoux, A. Buguin, and P. Silberzan, Velocity fields in a collectively migrating epithelium, Biophys. J. 98, 1790 (2010).

[8] M. Reffay, L. Petitjean, S. Coscoy, E. Grasland-Mongrain, F. Amblard, A. Buguin, and P. Silberzan, Orientation and polarity in collectively migrating cell structures: statics and dynamics, Biophys. J. 100, 2566 (2011).

[9] J. K. Klarlund, Dual modes of motility at the leading edge of migrating epithelial cell sheets, Proc. Natl. Acad. Sci. U.S.A. 109, 15799 (2012).

[10] M. Reffay, M. C. Parrini, O. Cochet-Escartin, B. Ladoux, A. Buguin, S. Coscoy, F. Amblard, J. Camonis, and P. Silberzan, Interplay of RhoA and mechanical forces in collective cell migration driven by leader cells, Nat. Cell Biol. 16, 217 (2014).

[11] M. Vishwakarma, J. Di Russo, D. Probst, U. S. Schwarz, T. Das, and J.P. Spatz, Mechanical interactions among followers determine the emergence of leaders in migrating epithelial cell collectives, Nat. Commun. 9, 3469 (2018).

[12] P. G. Saffman and G. Taylor, The penetration of a fluid into a porous medium or Hele-Shaw cell containing a more viscous liquid, Proc. R. Soc. A 245, 312 (1958). 
[13] J. Casademunt, Viscous fingering as a paradigm of interfacial pattern formation: Recent results and new challenges, Chaos 14, 809 (2004).

[14] N. Sepúlveda, L. Petitjean, O. Cochet, E. GraslandMongrain, P. Silberzan, and V. Hakim, Collective cell motion in an epithelial sheet can be quantitatively described by a stochastic interacting particle model, PLoS Comput. Biol. 9, e1002944 (2013).

[15] S. Mark, R. Shlomovitz, N. S. Gov, M. Poujade, E. Grasland-Mongrain, and P. Silberzan, Physical model of the dynamic instability in an expanding cell culture, Biophys. J. 98, 361 (2010).

[16] V. Tarle, A. Ravasio, V. Hakim, and N. Gov, Modeling the finger instability in an expanding cell monolayer, Integr. Biol. 7, 1218 (2015).

[17] M. Basan, J. Elgeti, E. Hannezo, W.-J. Rappel, and H. Levine, Alignment of cellular motility forces with tissue flow as a mechanism for efficient wound healing, Proc. Natl. Acad. Sci. U.S.A. 110, 2452 (2013).

[18] J. Zimmermann, M. Basan, and H. Levine, An instability at the edge of a tissue of collectively migrating cells can lead to finger formation during wound healing, Eur. Phys. J. Spec. Top. 223, 1259 (2014).

[19] D. Nesbitt, G. Pruessner, and C. F. Lee, Edge instability in incompressible planar active fluids, Phys. Rev. E 96, 062615 (2017).

[20] P. Lee and C. W. Wolgemuth, Crawling cells can close wounds without purse strings or signaling, PLoS Comput. Biol. 7, e1002007 (2011).

[21] A. Doostmohammadi, S. P. Thampi, T. B. Saw, C. T. Lim, B. Ladoux, and J. M. Yeomans, Celebrating soft matter's 10th anniversary: Cell division: a source of active stress in cellular monolayers, Soft Matter 11, 7328 (2015).

[22] D. L. Barton, S. Henkes, C. J. Weijer, and R. Sknepnek, Active Vertex Model for cell-resolution description of epithelial tissue mechanics, PLoS Comput. Biol. 13, e1005569 (2017).

[23] G. Y. Ouaknin and P. Z. Bar-Yoseph, Stochastic collective movement of cells and fingering morphology: no maverick cells, Biophys. J. 97, 1811 (2009).

[24] M. Salm and L. M. Pismen, Chemical and mechanical signaling in epithelial spreading, Phys. Biol. 9, 026009 (2012).

[25] M. H. Köpf and L. M. Pismen, A continuum model of epithelial spreading, Soft Matter 9, 3727 (2013).

[26] M. Ben Amar and C. Bianca, Onset of nonlinearity in a stochastic model for auto-chemotactic advancing epithelia, Sci. Rep. 6, 33849 (2016).

[27] C. Blanch-Mercader, R. Vincent, E. Bazellières, X. SerraPicamal, X. Trepat, and J. Casademunt, Effective viscosity and dynamics of spreading epithelia: a solvable model, Soft Matter 13, 1235 (2017).

[28] C. Pérez-González, R. Alert, C. Blanch-Mercader, M. GómezGonzález, T. Kolodziej, E. Bazellieres, J. Casademunt, and X. Trepat, Active wetting of epithelial tissues, Nat. Phys. 15, 79 (2019).

[29] R. Alert and J. Casademunt, Role of substrate stiffness in tissue spreading: wetting transition and tissue durotaxis, Langmuir, DOI: 10.1021/acs.langmuir.8b02037 (2018).
[30] X. Serra-Picamal, V. Conte, R. Vincent, E. Añón, D. T. Tambe, E. Bazellieres, J. P. Butler, J. J. Fredberg, and X. Trepat, Mechanical waves during tissue expansion, Nat. Phys. 8, 628 (2012).

[31] P. Recho, J. Ranft, and P. Marcq, One-dimensional collective migration of a proliferating cell monolayer, Soft Matter 12, 2381 (2016).

[32] S. Yabunaka and P. Marcq, Cell growth, division, and death in cohesive tissues: A thermodynamic approach, Phys. Rev. E 96, 022406 (2017).

[33] P.-G. de Gennes and J. Prost, The Physics of Liquid Crystals, 2nd ed. (Oxford University Press, New York, 1993).

[34] See Supplemental Material at http://link.aps.org/ supplemental/10.1103/PhysRevLett.122.088104 for a justification of the model and details of the linear stability analysis, which includes Refs. [35-60].

[35] R. Mayor and C. Carmona-Fontaine, Keeping in touch with contact inhibition of locomotion, Trends Cell Biol. 20, 319 (2010).

[36] B. Stramer and R. Mayor, Mechanisms and in vivo functions of contact inhibition of locomotion, Nat. Rev. Mol. Cell Biol. 18, 43 (2017).

[37] R. A. Desai, L. Gao, S. Raghavan, W. F. Liu, and C. S. Chen, Cell polarity triggered by cell-cell adhesion via Ecadherin, J. Cell Sci. 122, 905 (2009).

[38] A. A. Khalil and P. Friedl, Determinants of leader cells in collective cell migration, Integr. Biol. 2, 568 (2010).

[39] G. F. Weber, M. A. Bjerke, and D. W. DeSimone, A mechanoresponsive cadherin-keratin complex directs polarized protrusive behavior and collective cell migration, Dev. Cell 22, 104 (2012).

[40] E. Theveneau and R. Mayor, Collective cell migration of epithelial and mesenchymal cells, Cell Mol. Life Sci. 70, 3481 (2013).

[41] B. Ladoux, R.-M. Mège, and X. Trepat, Front-rear polarization by mechanical cues: from single cells to tissues, Trends Cell Biol. 26, 420 (2016).

[42] R. Mayor and S. Etienne-Manneville, The front and rear of collective cell migration, Nat. Rev. Mol. Cell Biol. 17, 97 (2016).

[43] J. Zimmermann, B. A. Camley, W.-J. Rappel, and H. Levine, Contact inhibition of locomotion determines cellcell and cell-substrate forces in tissues, Proc. Natl. Acad. Sci. U.S.A. 113, 2660 (2016).

[44] L. Coburn, H. Lopez, B. J. Caldwell, E. Moussa, C. Yap, R. Priya, A. Noppe, A. P. Roberts, V. Lobaskin, A. S. Yap, Z. Neufeld, and G. A. Gomez, Contact inhibition of locomotion and mechanical cross-talk between cell-cell and cell-substrate adhesion determine the pattern of junctional tension in epithelial cell aggregates, Mol. Biol. Cell 27, 3436 (2016).

[45] B. Smeets, R. Alert, J. Pešek, I. Pagonabarraga, H. Ramon, and R. Vincent, Emergent structures and dynamics of cell colonies by contact inhibition of locomotion, Proc. Natl. Acad. Sci. U.S.A. 113, 14621 (2016).

[46] R. Vincent, E. Bazellières, C. Pérez-González, M. Uroz, X. Serra-Picamal, and X. Trepat, Active Tensile Modulus of an Epithelial Monolayer, Phys. Rev. Lett. 115, 248103 (2015). 
[47] P. Marmottant, A. Mgharbel, J. Käfer, B. Audren, J.-P. Rieu, J.-C. Vial, B. van der Sanden, A. F. M. Marée, F. Graner, and H. Delanoë-Ayari, The role of fluctuations and stress on the effective viscosity of cell aggregates, Proc. Natl. Acad. Sci. U.S.A. 106, 17271 (2009).

[48] T. B. Saw, A. Doostmohammadi, V. Nier, L. Kocgozlu, S. Thampi, Y. Toyama, P. Marcq, C. T. Lim, J. M. Yeomans, and B. Ladoux, Topological defects in epithelia govern cell death and extrusion, Nature (London) 544, 212 (2017).

[49] K. Kruse, J. F. Joanny, F. Jülicher, J. Prost, and K. Sekimoto, Generic theory of active polar gels: a paradigm for cytoskeletal dynamics, Eur. Phys. J. E 16, 5 (2005).

[50] F. Jülicher, in New Trends in the Physics and Mechanics of Biological Systems, edited by M. Ben Amar, A. Goriely, M. M. Müller, and L. Cugliandolo (Oxford University Press, New York, 2011), Chap. 4.

[51] M. C. Marchetti, J. F. Joanny, S. Ramaswamy, T. B. Liverpool, J. Prost, M. Rao, and R. A. Simha, Hydrodynamics of soft active matter, Rev. Mod. Phys. 85, 1143 (2013).

[52] J. Prost, F. Jülicher, and J.-F. Joanny, Active gel physics, Nat. Phys. 11, 111 (2015).

[53] T. Wyatt, B. Baum, and G. Charras, A question of time: tissue adaptation to mechanical forces, Curr. Opin. Cell Biol. 38, 68 (2016).

[54] N. Khalilgharibi, J. Fouchard, P. Recho, G. Charras, and A. Kabla, The dynamic mechanical properties of cellularised aggregates, Curr. Opin. Cell Biol. 42, 113 (2016).

[55] J. Ranft, M. Basan, J. Elgeti, J.-F. Joanny, J. Prost, and F. Jülicher, Fluidization of tissues by cell division and apoptosis, Proc. Natl. Acad. Sci. U.S.A. 107, 20863 (2010).

[56] D. A. Matoz-Fernandez, K. Martens, R. Sknepnek, J. L. Barrat, and S. Henkes, Cell division and death inhibit glassy behaviour of confluent tissues, Soft Matter 13, 3205 (2017).

[57] R. Etournay, M. Popović, M. Merkel, A. Nandi, C. Blasse, B. Aigouy, H. Brandl, G. Myers, G. Salbreux, F. Jülicher, and S. Eaton, Interplay of cell dynamics and epithelial tension during morphogenesis of the Drosophila pupal wing, eLife 4, e07090 (2015).

[58] M. Krajnc, S. Dasgupta, P. Ziherl, and J. Prost, Fluidization of epithelial sheets by active cell rearrangements, Phys. Rev. E 98, 022409 (2018).

[59] F. Jülicher and J. Prost, Generic theory of colloidal transport, Eur. Phys. J. E 29, 27 (2009).

[60] C. Blanch-Mercader and J. Casademunt, Hydrodynamic instabilities, waves and turbulence in spreading epithelia, Soft Matter 13, 6913 (2017).

[61] D. Oriola, R. Alert, and J. Casademunt, Fluidization and Active Thinning by Molecular Kinetics in Active Gels, Phys. Rev. Lett. 118, 088002 (2017).

[62] P. Rosen and D.S. Misfeldt, Cell density determines epithelial migration in culture, Proc. Natl. Acad. Sci. U.S.A. 77, 4760 (1980).
[63] X. Trepat, M. R. Wasserman, T. E. Angelini, E. Millet, D. A. Weitz, J. P. Butler, and J. J. Fredberg, Physical forces during collective cell migration, Nat. Phys. 5, 426 (2009).

[64] O. Cochet-Escartin, J. Ranft, P. Silberzan, and P. Marcq, Border forces and friction control epithelial closure dynamics, Biophys. J. 106, 65 (2014).

[65] R. A. Foty, G. Forgacs, C. M. Pfleger, and M. S. Steinberg, Liquid Properties Of Embryonic Tissues: Measurement of Interfacial Tensions, Phys. Rev. Lett. 72, 2298 (1994).

[66] G. Forgacs, R. A. Foty, Y. Shafrir, and M. S. Steinberg, Viscoelastic properties of living embryonic tissues: a quantitative study, Biophys. J. 74, 2227 (1998).

[67] K. Guevorkian, M.-J. Colbert, M. Durth, S. Dufour, and F. Brochard-Wyart, Aspiration of Biological Viscoelastic Drops, Phys. Rev. Lett. 104, 218101 (2010).

[68] T. V. Stirbat, A. Mgharbel, S. Bodennec, K. Ferri, H. C. Mertani, J.-P. Rieu, and H. Delanoë-Ayari, Fine tuning of tissues' viscosity and surface tension through contractility suggests a new role for $\alpha$-catenin, PLoS One 8, e52554 (2013).

[69] J. Engmann, C. Servais, and A. S. Burbidge, Squeeze flow theory and applications to rheometry: A review, J. Nonnewton. Fluid Mech. 132, 1 (2005).

[70] S. M. Troian, E. Herbolzheimer, S. A. Safran, and J. F. Joanny, Fingering instabilities of driven spreading films, Europhys. Lett. 10, 25 (1989).

[71] F. Melo, J. F. Joanny, and S. Fauve, Fingering Instability of Spinning Drops, Phys. Rev. Lett. 63, 1958 (1989).

[72] M. Ben Amar and L. J. Cummings, Fingering instabilities in driven thin nematic films, Phys. Fluids 13, 1160 (2001).

[73] A. C. Callan-Jones, J.-F. Joanny, and J. Prost, ViscousFingering-Like Instability of Cell Fragments, Phys. Rev. Lett. 100, 258106 (2008).

[74] S. Sankararaman and S. Ramaswamy, Instabilities and Waves in Thin Films of Living Fluids, Phys. Rev. Lett. 102, 118107 (2009).

[75] N. Sarkar and A. Basu, Instabilities and diffusion in a hydrodynamic model of a fluid membrane coupled to a thin active fluid layer, Eur. Phys. J. E 35, 115 (2012).

[76] N. Sarkar and A. Basu, Generic instabilities in a fluid membrane coupled to a thin layer of ordered active polar fluid, Eur. Phys. J. E 36, 86 (2013).

[77] A. Nagilla, R. Prabhakar, and S. Jadhav, Linear stability of an active fluid interface, Phys. Fluids 30, 022109 (2018).

[78] J. J. Williamson and G. Salbreux, Stability and Roughness of Interfaces in Mechanically Regulated Tissues, Phys. Rev. Lett. 121, 238102 (2018).

[79] M. Bogdan and T. Savin, Fingering instabilities in tissue invasion: an active fluid model, R. Soc. Open Sci. 5, 181579 (2018). 